\title{
Silencing of kallikrein-related peptidase 6 attenuates the proliferation, migration, and invasion of gastric cancer cells through inhibition of epithelial-mesenchymal transition
}

\author{
DONG ZHOU, YANPING HE, HENGPING LI and WEIDONG HUANG
}

\author{
Department of Vascular Surgery, No. 1 People's Hospital, Hubei University of Medicine, \\ Xiangyang, Hubei 441000, P.R. China
}

Received July 31, 2020; Accepted March 17, 2021

DOI: $10.3892 / \mathrm{etm} .2021 .10202$

\begin{abstract}
Kallikrein-related peptidase 6 (KLK6), a member of the kallikrein-related peptidase family, is involved in the regulation of epithelial-mesenchymal transition (EMT) in cancer cells and is highly expressed in gastric cancer tissues. The aim of the present study was to investigate the effect of KLK6 on the proliferation, migration and invasion of gastric cancer cells and to determine the underlying mechanism of its actions. The expression of KLK6 was measured in metastatic gastric cancer cells using western blotting and reverse transcription-quantitative PCR, and KLK6 was overexpressed or inhibited in HGC-27 cells using plasmid transfection. Cell proliferation, migration, invasion and EMT were also evaluated using Cell Counting Kit 8, Transwell and western blot analysis, respectively. In addition, a mouse xenograft model was constructed by injection of HGC-27 cells. The xenograft was treated with KLK6 interference or overexpression plasmids to study the in vivo effects of KLK6 on tumor development. The results demonstrated that KLK6 was highly expressed in HGC-27 cells and that KLK6 inhibition attenuated cell proliferation, migration and invasion and prevented gastric cancer tumor development. In addition, KLK6 inhibition reduced the expression of epithelial cell adhesion molecule and vimentin, reduced the phosphorylation of SMAD2 and SMAD3 and upregulated epithelial-cadherin expression. In conclusion, KLK6 inhibition suppressed the proliferation, migration and invasion of gastric cancer cells both in vitro and in vivo through the inhibition of EMT. These findings indicate that KLK6 a potential therapeutic target for gastric cancer therapy.
\end{abstract}

Correspondence to: Dr Dong Zhou, Department of Vascular Surgery, No. 1 People's Hospital, Hubei University of Medicine, 15 Jiefang Road, Fancheng, Xiangyang, Hubei 441000, P.R. China E-mail: tbw120@163.com

Key words: gastric cancer, kallikrein-related peptidase 6, migration, invasion, epithelial-mesenchymal transition

\section{Introduction}

Gastric cancer is one of the most prevalent malignant tumors worldwide (1). The number of newly diagnosed cases of gastric cancer increased to 1.033 million worldwide in 2018, with 782,000 related deaths (2). With aging and socioeconomic development, cancer morbidity and mortality are rapidly increasing globally (3). A variety of advanced methods, including chemotherapy, surgical removal and radiotherapy, have been used for gastric cancer treatment, however, mortality remains high (4). An estimated 456,000 new cases of gastric cancer were diagnosed in China in 2018, with approximately 390,000 related deaths (5). Therefore, it is imperative to develop effective therapeutic strategies for gastric cancer treatment and gene therapy is a potentially beneficial option (6).

Kallikrein-related peptidase 6 (KLK6) is a member of the KLK family that was initially identified based on its abnormal expression in human ovarian and breast cancers (7). Accumulating evidence has demonstrated the role of KLK6 in carcinogenesis and its potential as a cancer biomarker (8). As a proteolytic protein, KLK6 regulates the invasive phenotype of tumor cells by degrading fibronectin, fibrinogen, collagen and laminin $(9,10)$. Previous research indicated that KLK6 promoted cancer cell migration and invasion by modulating epithelial-mesenchymal transition (EMT) (11). However, the effect of KLK6 on EMT varies based on tumor classification. KLK6 expression in non-KL6 expressing breast cancer cells downregulated the expression of the EMT marker vimentin and upregulated the expression of the epithelial markers cytokeratin 8 and 19, suggesting an inhibitory effect of KLK6 against EMT in breast cancer cells (12). Conversely, KLK6 overexpression or expression in KLK6-knockdown colorectal cancer cells facilitated EMT (11).

Previous studies have demonstrated that KLK6 is upregulated in gastric cancer (13-15). However, the effect of KLK6 on the invasive phenotype and EMT status of gastric cancer cells requires further study. In the present study KLK6 was expressed at significantly higher levels in metastatic gastric cancer cells (HGC-27) than in primary gastric cancer cells (AGS and SNU-1). Subsequently, the effect of KLK6 on the 
invasive phenotype and EMT status of gastric cancer cells lines HGC-27 in vitro and in vivo in a mouse xenograft model were investigated.

\section{Materials and methods}

Cell culture. Human gastric cancer cell line HGC-27 (derived from a lymphatic metastasis) and primary gastric cancer cell lines AGS and SNU-1 were purchased from The Cell Bank of Type Culture Collection of The Chinese Academy of Sciences. The cells were incubated in RPMI-1640 medium (Cytiva; cat. no. SH30809.01B) containing 10\% fetal bovine serum (FBS; Gibco; Thermo Fisher Scientific, Inc.; cat. no. 10270-106) at $37^{\circ} \mathrm{C}$ in an environment containing $5 \% \mathrm{CO}_{2}$. The protein and mRNA expression levels of KLK6 in HGC-27, AGS and SNU-1 cells were measured using western blotting and reverse transcription-quantitative polymerase chain reaction (RT-qPCR), respectively.

Cell transfection.pSICOR interference vectors and pCDH-CMVMCS-EF1-CopGFP-T2A-Puro overexpression vectors were supplied by Addgene, Inc. KLK6 mRNA was amplified via PCR(forward,5'-GCTCTAGATGAAGAAGCTGATGGTG-3'; reverse, 5'-CGGGATCCTCACTTGGCCTGAATGGT-3') and inserted into the $\mathrm{pCDH}$ vector to produce $\mathrm{pCDH-KLK6}$ overexpression (OV) plasmids. The short hairpin (sh) interference fragments (shKLK6-1, 5'-AGAATAAGTTGGTGCATGG-3'; shKLK6-2, 5'-CAGATGGTGATTTCCCTGAC-3'; shKLK6-3, 5'-GATCAAAGGAGAAGCCAGGA-3'; and shKLK6-4, 5'-CAGATACACGAACTGGATCC-3') were inserted into pSICOR vectors to produce pSICOR-shKLK6 plasmids. HGC-27 cells were transfected for $48 \mathrm{~h}$ with $0.8 \mu \mathrm{g}$ pCDH-KLK6 (OV-KLK6), pSICOR-shKLK6 (shRNA) or their corresponding negative controls (OV-NC and sh-NC, respectively) using Lipofectamine ${ }^{\circledR} 2000$ (Invitrogen; Thermo Fisher Scientific, Inc.; cat. no. 11668-027). Non-transfected cells served as the control (CON).

3-(4,5-Dimethylthiazol-2-yl)-2,5-diphenyltetrazolium bromide (MTT) assay. Cell viability was measured using the MTT assay. HGC-27 cells were seeded in a 96-well plate at a density of $5 \times 10^{3}$ cells/well and maintained overnight at $37^{\circ} \mathrm{C}$ with $5 \% \mathrm{CO}_{2}$. A $20 \mu 1$ volume of MTT reagent (Bioswamp Life Science Lab; Wuhan Beinlai Biotechnology Co., Ltd.; cat. no. C1736) was added to each well after 24,48 and $72 \mathrm{~h}$ of transfection. The cells were incubated at $37^{\circ} \mathrm{C}$ for $4 \mathrm{~h}$ in an atmosphere containing $5 \% \mathrm{CO}_{2}$ and subsequently incubated with $150 \mu 1$ of dimethyl sulfoxide for $10 \mathrm{~min}$. The absorbance of the wells was measured using a microplate reader at $570 \mathrm{~nm}$.

Flow cytometry. Flow cytometry was performed to evaluate apoptosis and cell cycle progression in HGC-27 cells. To assess cell apoptosis, $1 \times 10^{6}$ HGC-27 cells were resuspended in $1 \mathrm{ml}$ of phosphate-buffered saline (PBS) and centrifuged at $400 \mathrm{x} \mathrm{g}$ for $5 \mathrm{~min}$ at $4^{\circ} \mathrm{C}$. The cells were then resuspended in $200 \mu \mathrm{l}$ of PBS, stained with $10 \mu \mathrm{l}$ of Annexin V-fluorescein isothiocyanate (BD Biosciences), and $10 \mu \mathrm{l}$ of propidium iodide (PI; BD Biosciences) in the dark at $4^{\circ} \mathrm{C}$ for $30 \mathrm{~min}$. Thereafter, the cells were subjected to flow cytometry. To assess cell cycle progression, $1 \times 10^{7} \mathrm{HGC}-27$ cells were fixed in a mixture of $300 \mu \mathrm{l}$ of PBS and $700 \mu \mathrm{l}$ of absolute ethyl alcohol at $-20^{\circ} \mathrm{C}$ for $24 \mathrm{~h}$. After two washes with PBS, the cells were resuspended in $100 \mu \mathrm{l}$ of RNase A (BD Biosciences) and maintained at $37^{\circ} \mathrm{C}$ for $30 \mathrm{~min}$. The cells were then stained with $400 \mu \mathrm{l}$ of PI $(50 \mu \mathrm{g} / \mathrm{ml})$ in the dark at $4^{\circ} \mathrm{C}$ for $5 \mathrm{~min}$ and subjected to flow cytometry (NovoCyte, ACEA Biosciences, Inc.; Agilent Technologies, Inc.). The data were analyzed using NovoExpress software version 1.3.0 (ACEA Biosciences, Inc.; Agilent Technologies, Inc.). For apoptosis analysis, both earlyand late-stage apoptosis (quadrants Q2-2 and Q2-4) were evaluated.

Wound healing. HGC-27 cells were cultured overnight in 6-well plates at a density of $1 \times 10^{6}$ cells/well. After $4 \mathrm{~h}$ of transfection, the cells were wounded by scratching the cell monolayer with a sterile $200-\mu 1$ plastic pipette tip. The scratches were observed and imaged under an inverted fluorescence microscope (magnification, x100; Leica Microsystems, $\mathrm{GmbH}$ ) after incubation in serum-free medium at $37^{\circ} \mathrm{C}$ in an environment containing $5 \% \mathrm{CO}_{2}$ for 0 and $24 \mathrm{~h}$.

Cell migration and invasion assays. Cell migration and invasion were evaluated using two-chamber Transwell inserts (Corning, Inc.). The cells were starved in serum-free medium for $24 \mathrm{~h}$ and resuspended in RPMI-1640 medium (Cytiva; cat. no. SH30809.01B) containing 1\% FBS. In the upper chambers, $0.5 \mathrm{ml}$ of treated cells were seeded at a density of $1 \times 10^{5}$ cells $/ \mathrm{ml}$ and the lower chambers were filled with $0.75 \mathrm{ml}$ of RPMI-1640 medium supplemented with 10\% FBS. The inserts for the cell invasion assay were pre-coated for $30 \mathrm{~min}$ at $37^{\circ} \mathrm{C}$ with Matrigel (BD Biosciences; cat. no. 354230) between the lower and upper chambers. After $24 \mathrm{~h}$ of incubation at $37^{\circ} \mathrm{C}$, the cells were fixed at $25^{\circ} \mathrm{C}$ with $4 \%$ paraformaldehyde for $10 \mathrm{~min}$ and stained with $1 \mathrm{ml}$ of $0.5 \%$ crystal violet (Life Science Lab; Wuhan Beinlai Biotechnology Co., Ltd.; cat. no. C1701) for $30 \mathrm{~min}$ at room temperature. Non-invading or non-migrating cells were wiped away using cotton swabs, and the migrated or invaded cells were counted under an inverted fluorescence microscope (magnification, x100; Leica Microsystems $\mathrm{GmbH}$ ).

$R T-q P C R$. RT-qPCR was performed to examine the mRNA expression levels of KLK6 in HGC-27, AGS and SNU-1 cells, as well as EMT-related genes in HGC-27 cells. Total RNA was extracted from HGC-27, AGS and SNU-1 cells using TRIzol ${ }^{\circledR}$ reagent (Thermo Fisher Scientific, Inc.) and reverse-transcribed into cDNA using the M-MuLV kit $\left(42^{\circ} \mathrm{C}\right.$ for $60 \mathrm{~min} ; 70^{\circ} \mathrm{C}$ for $15 \mathrm{~min}$ and held at $16^{\circ} \mathrm{C}$ ), according to the manufacturer's instructions (Takara Bio, Inc). The collected cDNA was amplified using a SYBR Green PCR kit (KAPA Biosystems, Inc.; Roche Diagnostics; cat. no. KM4101) following the manufacturer's instructions in a CFX-Connect 96 apparatus (Bio-Rad Laboratories, Inc.). The thermocycling conditions were as follows: $95^{\circ} \mathrm{C}$ for $3 \mathrm{~min} ; 39$ cycles of denaturation at $95^{\circ} \mathrm{C}$ for $5 \mathrm{sec}$, annealing at $56^{\circ} \mathrm{C}$ for $10 \mathrm{sec}$, and extension at $72^{\circ} \mathrm{C}$ for $25 \mathrm{sec}$; and final extension at $65^{\circ} \mathrm{C}$ for $5 \mathrm{sec}$ and $95^{\circ} \mathrm{C}$ for $50 \mathrm{sec}$. The primer sequences were as follows: KLK6 forward, 5'-GAACTCATCCAGCCCCTT-3' and reverse, 5'-CATCCCC AGCACACAACA-3'; epithelial (E)-cadherin forward, 5'-GGC 
AAGGTTTTCTACAGC-3' and reverse, 5'-ATGTGGCA ATGCGTTCT-3'; vimentin forward, 5'-TTGAACGCAAAGT GGAATC-3'; and reverse, 5'-AGGTCAGGCTTGGAAACA-3'; GAPDH forward, 5'-CCACTCCTCCACCTTTG-3' and reverse, 5'-CACCACCCTGTTGCTGT-3'. GAPDH served as an endogenous control. Relative mRNA expression levels were calculated using the $2^{-\Delta \Delta \mathrm{Cq}}$ method (16).

Western blotting. Western blotting was performed to determine the protein expression levels of KLK6 in HGC-27, AGS and SNU-1 cells and EMT-related markers in HGC-27 cells. Total proteins were collected using radioimmunoprecipitation assay lysis buffer (Bioswamp Life Science Lab; Wuhan Beinlai Biotechnology Co., Ltd.; cat. no. W1689) and quantified using a bicinchoninic acid assay kit (Bioswamp Life Science Lab; Wuhan Beinlai Biotechnology Co., Ltd.; cat. no. W1712). Proteins $(20 \mu \mathrm{g})$ were separated by $12 \%$ sodium dodecyl sulfate-polyacrylamide gel electrophoresis and transferred onto polyvinylidene fluoride membranes (EMD Millipore). The membranes were blocked and incubated overnight at $4^{\circ} \mathrm{C}$ with primary antibodies against epithelial cell adhesion molecules (EP-CAM, Bioswamp, cat. no. PAB30814, 1:1000), E-cadherin (Bioswamp Life Science Lab; Wuhan Beinlai Biotechnology Co., Ltd.; cat. no. PAB43792, 1:1000), vimentin (Bioswamp Life Science Lab; Wuhan Beinlai Biotechnology Co., Ltd; cat. no. PAB40646; 1:1,000), phosphorylated (p)-SMAD2 (Bioswamp Life Science Lab; Wuhan Beinlai Biotechnology Co., Ltd; cat. no. PAB43294-P; 1:1,000), SMAD2 (Bioswamp Life Science Lab; Wuhan Beinlai Biotechnology Co., Ltd; cat. no. PAB30712; 1:1,000), p-SMAD3 (Bioswamp Life Science Lab; Wuhan Beinlai Biotechnology Co., Ltd; cat. no. PAB43521-P; 1:1,000), SMAD3 (Bioswamp Life Science Lab; Wuhan Beinlai Biotechnology Co., Ltd; cat. no. PAB30705; 1:1,000), and GAPDH (Bioswamp Life Science Lab; Wuhan Beinlai Biotechnology Co., Ltd; cat. no. PAB36269; 1:1,000) and were subsequently incubated for $1 \mathrm{~h}$ at room temperature with HRP-conjugated goat anti-rabbit IgG secondary antibodies (Bioswamp Life Science Lab; Wuhan Beinlai Biotechnology Co., Ltd; cat. no. SAB43714; 1:20,000). The membranes were developed using ECL (EMD Millipore) and then observed using a Tanon-5200 apparatus (Tanon Science \& Technology Co., Ltd.) and data were analyzed using Tanon GIS software version 4.2 (Tanon Science \& Technology Co., Ltd.). GAPDH served as an internal reference.

Tumor xenografts in nude mice. Female BALB/c nude mice (age, 6 weeks; weight 18-20 g; $\mathrm{n}=15$ ) were supplied by Changzhou Cavens Experimental Animal Co. Ltd. (ref. no. 1107301911000025) and divided into five groups ( $\mathrm{n}=3$ per group): CON, sh-NC, sh-KLK6, OV-NC and OV-KLK6. All mice were injected with $200 \mu 1$ of HGC-27 cells at a density of $1 \times 10^{6}$ cells $/ \mathrm{m}$ in the right axilla. When the tumor size reached $80-100 \mathrm{~mm}^{3}$ (12 days after injection), the mice were treated with $10 \mu \mathrm{g}$ of PBS, sh-NC, sh-KLK6, OV-NC and OV-KLK6, respectively, by intratumoral injections once every three days for a total of four times. Animal health and behavior were monitored every day. When the tumor could be clearly identified (4 days after injection), tumor volume was measured every two days using the following formula: volume $\left(\mathrm{mm}^{3}\right)=$ length $\mathrm{x}$ width $2 / 2$ (17). After 10 days of treatment, the mice were euthanized with an intraperitoneal injection of sodium pentobarbital at $100 \mathrm{mg} / \mathrm{kg}$ of body weight (death was confirmed by the absence of heartbeat and breath), and the tumors were removed for further analysis. The humane endpoint was when the tumor maximum diameter reached $>15 \mathrm{~mm}$. No animals died during the course of the experiment. All animal procedures were approved by the ethics committee of Wuhan Myhalic Biotechnology Co., Ltd. (approval no. HLK-20181102-01), who also conducted the animal experiments.

Hematoxylin and eosin $(H \& E)$ staining. Pathological changes in the tumors were evaluated by H\&E staining. The tissues were fixed in $10 \%$ formalin buffer at $25^{\circ} \mathrm{C}$ for $48 \mathrm{~h}$, embedded in paraffin and sectioned at a thickness of $4 \mu \mathrm{m}$. After dewaxing, the sections were stained with hematoxylin (Bioswamp, cat. no. I1709) at $25^{\circ} \mathrm{C}$ for $3 \mathrm{~min}$ and then stained with eosin solution (Bioswamp Life Science Lab; Wuhan Beinlai Biotechnology Co., Ltd; cat. no. I1703) for $3 \mathrm{~min}$. Pathological changes were assessed using a light microscope (magnification, x100), Leica Microsystems GmbH).

Statistical analysis. Data are presented as the mean \pm standard deviation (SD). Differences between two groups were analyzed using unpaired Student's t-test and those between more than two groups were analyzed using one-way analysis of variance, followed by Tukey's test. $\mathrm{P}<0.05$ was considered statistically significant.

\section{Results}

KLK6 is highly expressed in $\mathrm{HGC}-27$ cells and increases $H G C-27$ cell viability. As indicated in Fig. $1 \mathrm{~A}$ and $\mathrm{B}$, the expression of KLK6 was significantly higher in metastatic gastric cancer cells (HGC-27) than in primary gastric cancer cells (AGS and SNU-1). Thus, the effect of KLK6 on gastric cancer was investigated in vitro and in vivo using HGC-27 cells. In HGC-27 cells, KLK6 was inhibited by pSICOR-shKLK6 plasmids and overexpressed by pCDH-KLK6 plasmids (Fig. 1C-F). As shRNA3 interference resulted in the greatest reduction in the expression of KLK6, it was selected for subsequent experiments. MTT assay demonstrated that KLK6 overexpression enhanced the viability of HGC-27 cells, whereas KLK6 inhibition attenuated cell viability in a time-dependent manner in comparison with controls (Fig. 2A). After $24 \mathrm{~h}$ of transfection, cell viability was significantly different between the CON group and the sh-KLK6 or OV-KLK6 groups. Therefore, subsequent experiments were performed $24 \mathrm{~h}$ after transfection. Flow cytometry indicated that KLK6 inhibition promoted apoptosis and G2/M phase arrest in HGC-27 cells, while KLK6 overexpression showed the opposite effect (Fig. 2B).

KLK6 inhibition attenuates $H G C-27$ cell migration and invasion. The wound healing ability of sh-KLK6-transfected HGC-27 cells was poorer than that of control cells, whereas it was increased by KLK6 overexpression (Fig. 3A). In addition, the Transwell assay showed that KLK6 inhibition reduced the number of migratory and invading cells in comparison with 
A
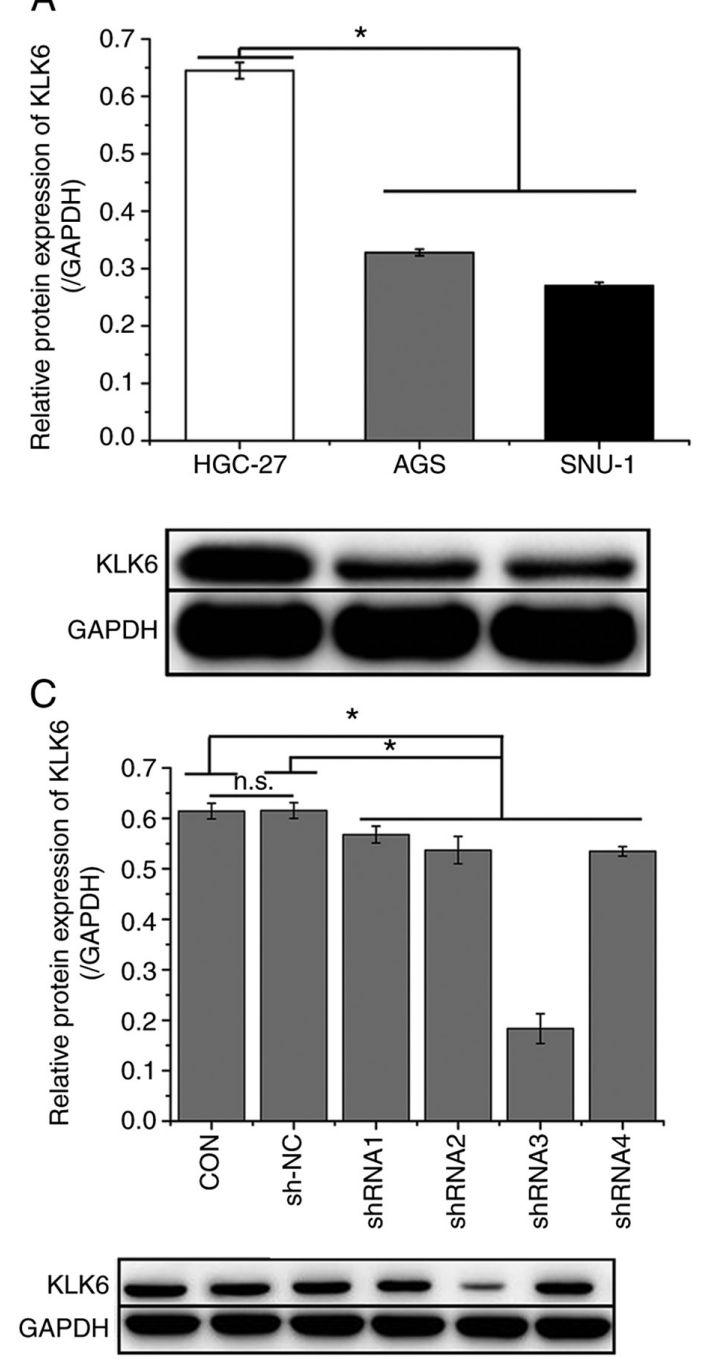

E
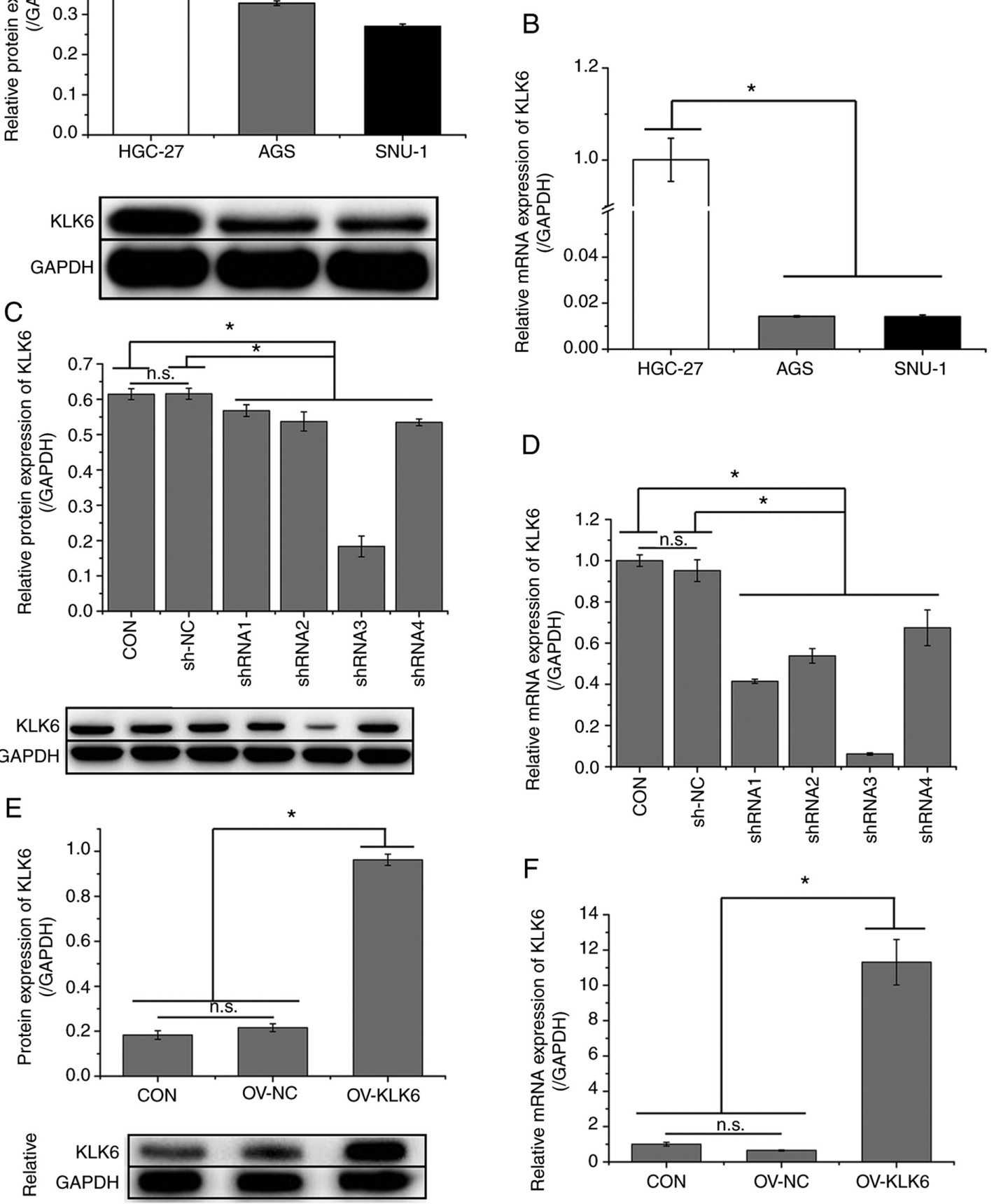

Figure 1. KLK6 is highly expressed in HGC-27 cells. Relative (A) protein and (B) mRNA expression levels of KLK6 in HGC-27, AGS and SNU-1 cells. Relative (C) protein and (D) mRNA expression levels of KLK6 in HGC-27 cells after plasmid transfection for $24 \mathrm{~h}$. Relative (E) protein and (F) mRNA expression of KLK6 in HGC-27 cells after overexpression plasmid transfection for $24 \mathrm{~h}$. Data are presented as the mean \pm SD ( $=3$ ). KLK6, kallikrein-related peptidase 6; Con, untreated control; shRNA, short hairpin RNA; NC, non-coding control; OV, overexpression plasmid; n.s., not significant. ${ }^{*} \mathrm{P}<0.05$.

the control, whereas numbers were increased by KLK6 overexpression (Fig. 3B and C).

KLK6 is involved in EMT regulation in HGC-27 cells. Western blotting and RT-qPCR were performed to evaluate the expression of EMT-related factors. As shown in Fig. 4, compared to the CON group, the protein and mRNA expression levels of E-cadherin were increased by sh-KLK6 but decreased by OV-KLK6 in comparison with controls, whereas vimentin showed the opposite trend as that of E-cadherin. 

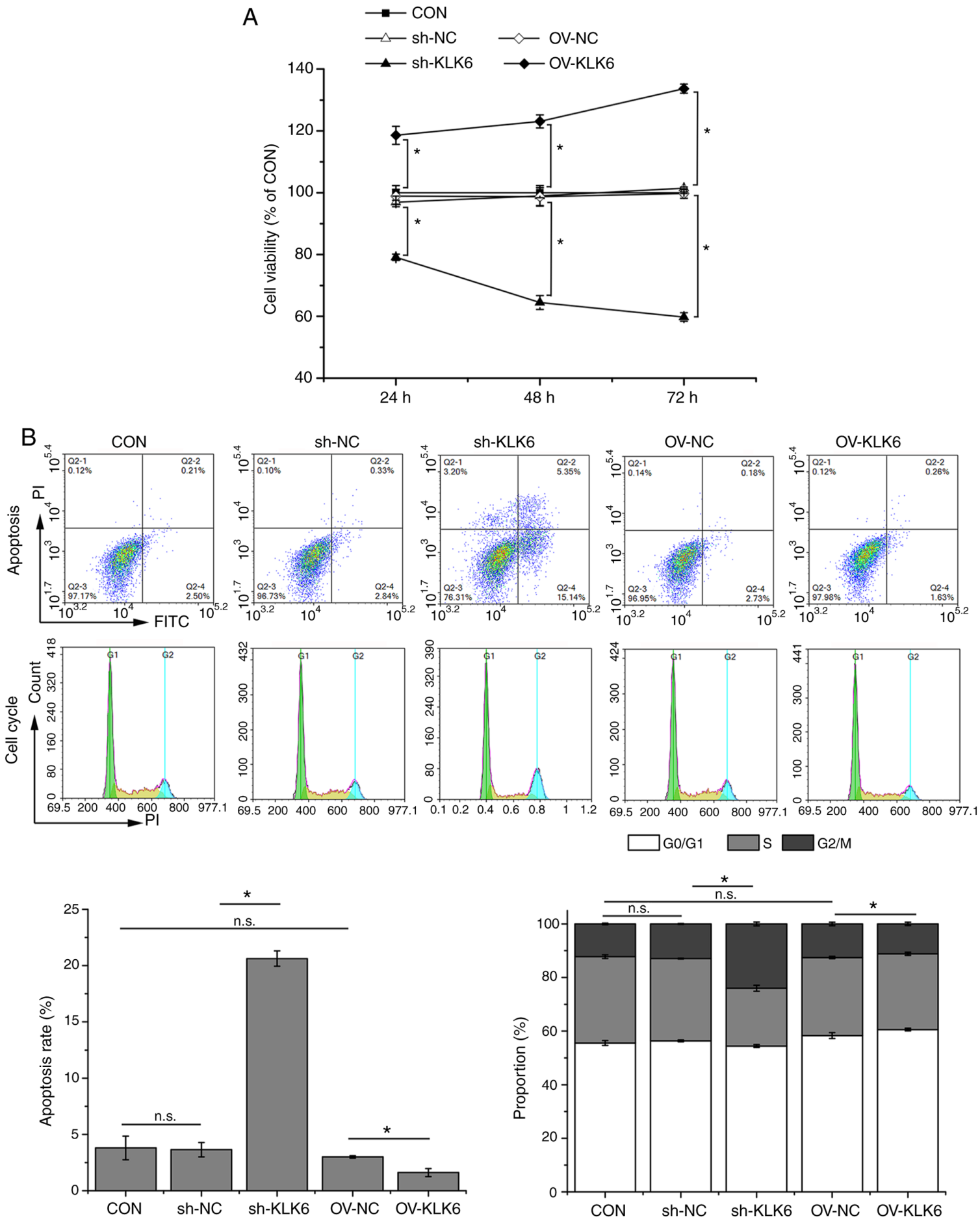

Figure 2. KLK6 inhibition suppresses viability and increases apoptosis of HGC-27 cells. (A) HGC-27 cell viability after 24, 48, and 72 h of transfection. (B) Apoptosis rate and cell cycle stage of HGC-27 cells. Data are presented as the mean \pm SD ( $n=3$ ). KLK6, kallikrein-related peptidase 6; Con, untreated control; sh, short hairpin; NC, non-coding control; OV, overexpression plasmid; n.s., not significant. " $\mathrm{P}<0.05$.

Additionally, KLK6 inhibition suppressed the protein expression of EP-CAM and the phosphorylation of SMAD2 and SMAD3. These results demonstrated the regulatory effect of KLK6 on EMT in HGC-27 cells through modulation of EMT-related proteins.

KLK6 inhibition suppresses gastric cancer development in vivo. To investigate the effect of KLK6 on gastric cancer in vivo, a xenograft mouse model was constructed by injecting
HGC-27 cells. Mice were treated with sh-KLK6, OV-KLK6 or the corresponding negative controls. The results demonstrated that both the mRNA and protein expression levels of KLK6 in tumor tissues were increased compared to those in normal tissues (Fig. 5). KLK6 overexpression promoted the growth of gastric cancer, while KLK6 inhibition blocked tumor growth (Fig. 6A-C). H\&E staining revealed that KLK6 increased tumor progression (Fig. 6D). In addition, the expression of EMT-related proteins in tumors was measured. Compared 
A
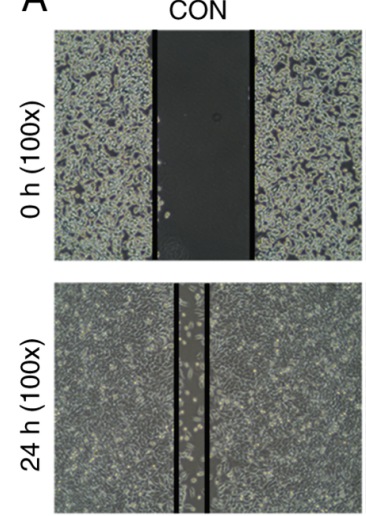

B
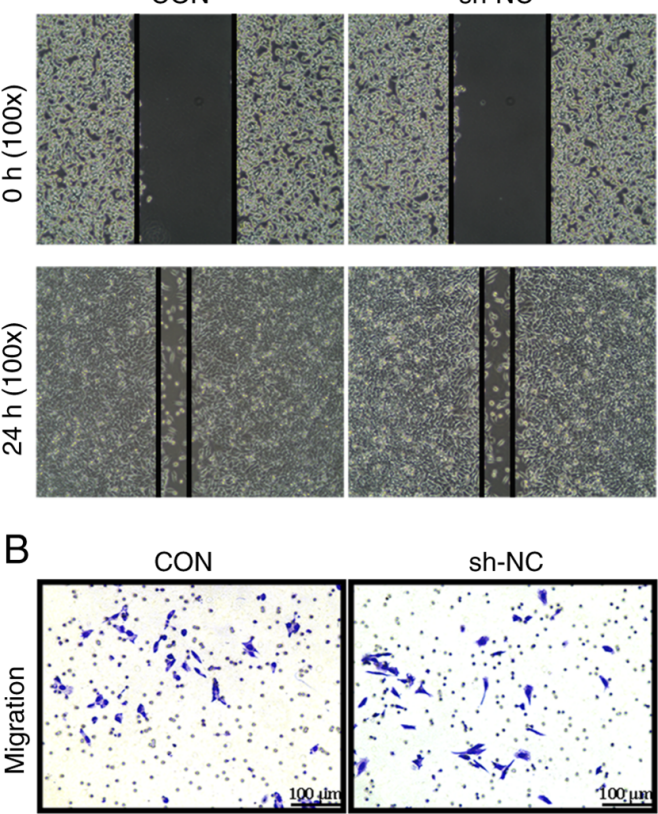

sh-KLK6

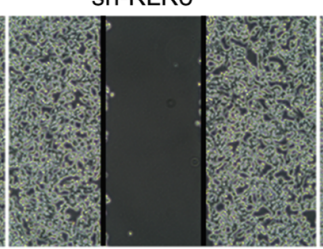

OV-NC
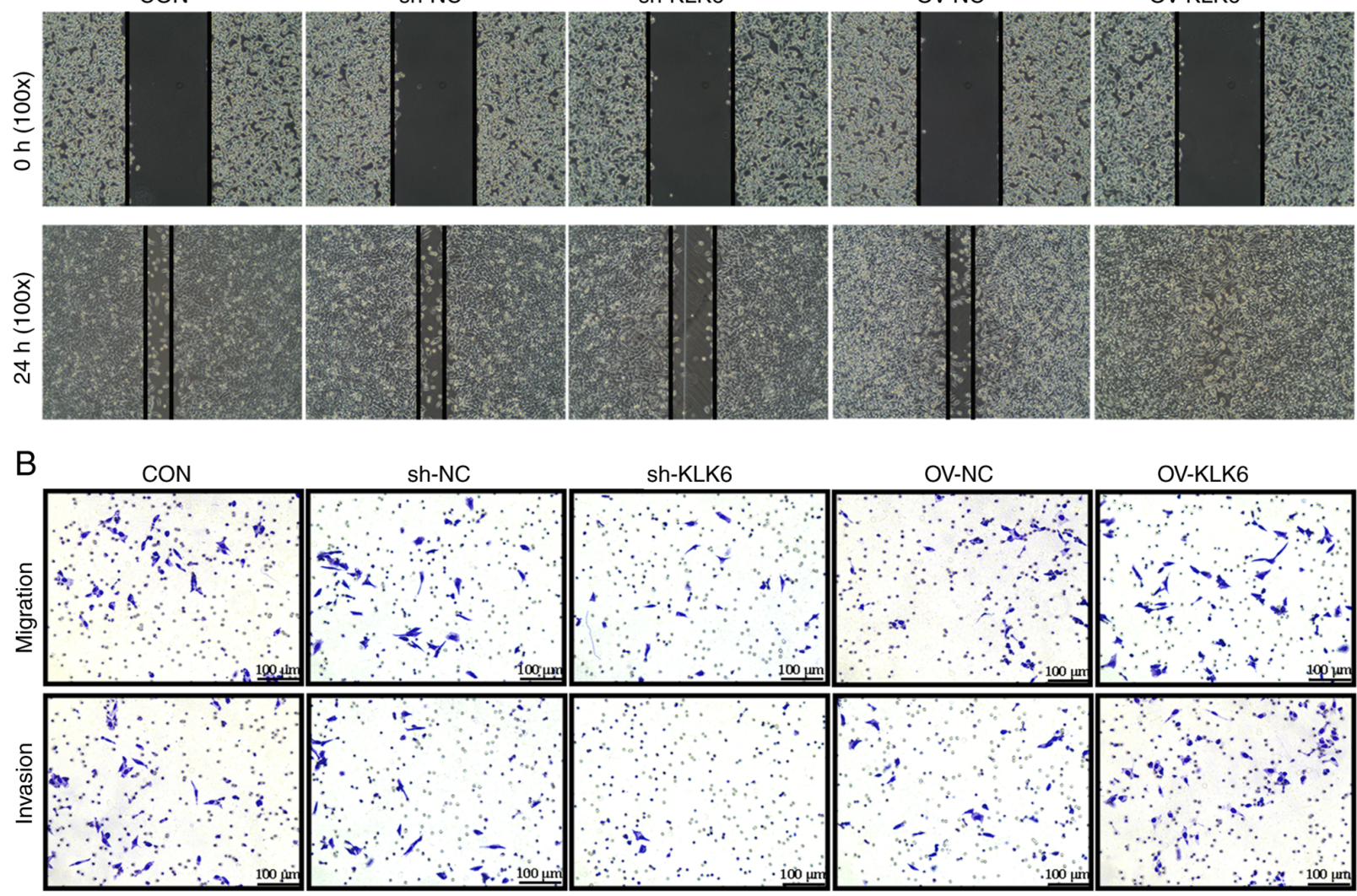

C
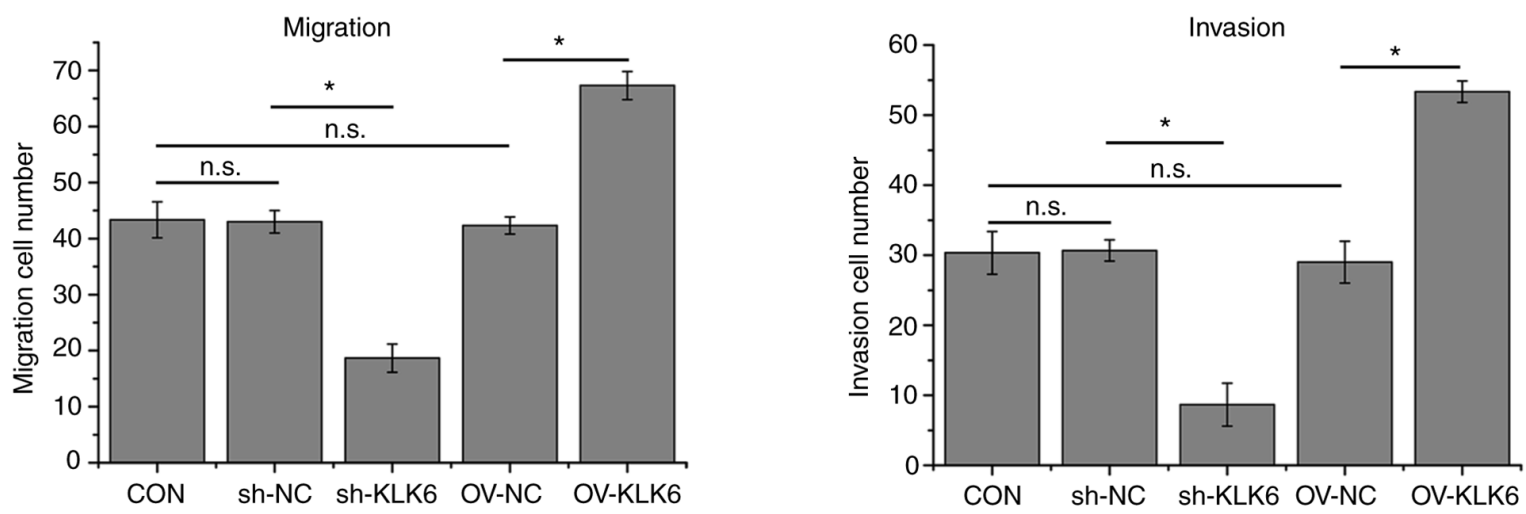

Figure 3. KLK6 inhibition attenuates HGC-27 cell migration and invasion. (A) Wound healing of HGC-27 cells. (B) Migration and invasion of HGC-27 cells (C) Quantification of cell migration and invasion. Data are presented as the mean \pm SD ( $=3$ ). KLK6, kallikrein-related peptidase 6; Con, untreated control; sh, short hairpin; NC, non-coding control; OV, overexpression plasmid; n.s., not significant. " $\mathrm{P}<0.05$.

to the $\mathrm{CON}$ and negative controls, the protein expression of EP-CAM and vimentin and the phosphorylation of SMAD2, and SMAD3 was downregulated by sh-KLK6, whereas that of E-cadherin was upregulated, with OV-KLK6 showing the opposite trend (Fig. 6E).

\section{Discussion}

In the present study it was demonstrated that KLK6 was expressed at significantly higher levels in metastatic gastric cancer cells (HGC-27) than in primary gastric cancer cells (AGS and SNU-1). KLK6 interference inhibited the proliferation, migration and invasion of gastric cancer cells in vitro and suppressed the growth of gastric cancer cell line tumors in vivo. In addition, KLK6 interference attenuated EMT in gastric cancer cells by regulating the expression and phosphorylation of EMT-related proteins (E-cadherin, vimentin, EP-CAM, SMAD2 and SMAD3). KLKs are a family of serine proteases that contains 15 members, which are associated with various physiological functions (18). Several KLKs, including KLK6, are involved in neoplastic malignant progression and transformation, through regulation of tumor chemoresistance, migration, invasion and growth $(18,19)$. Aberrant expression of KLK6 is common in a number of malignancies, including head and neck squamous cell carcinoma (20) and colorectal (21), ovarian (22) and melanoma skin cancer (23). KLK6 is highly expressed in patients with head and neck squamous cell carcinoma and modulates cancer cell migration, 

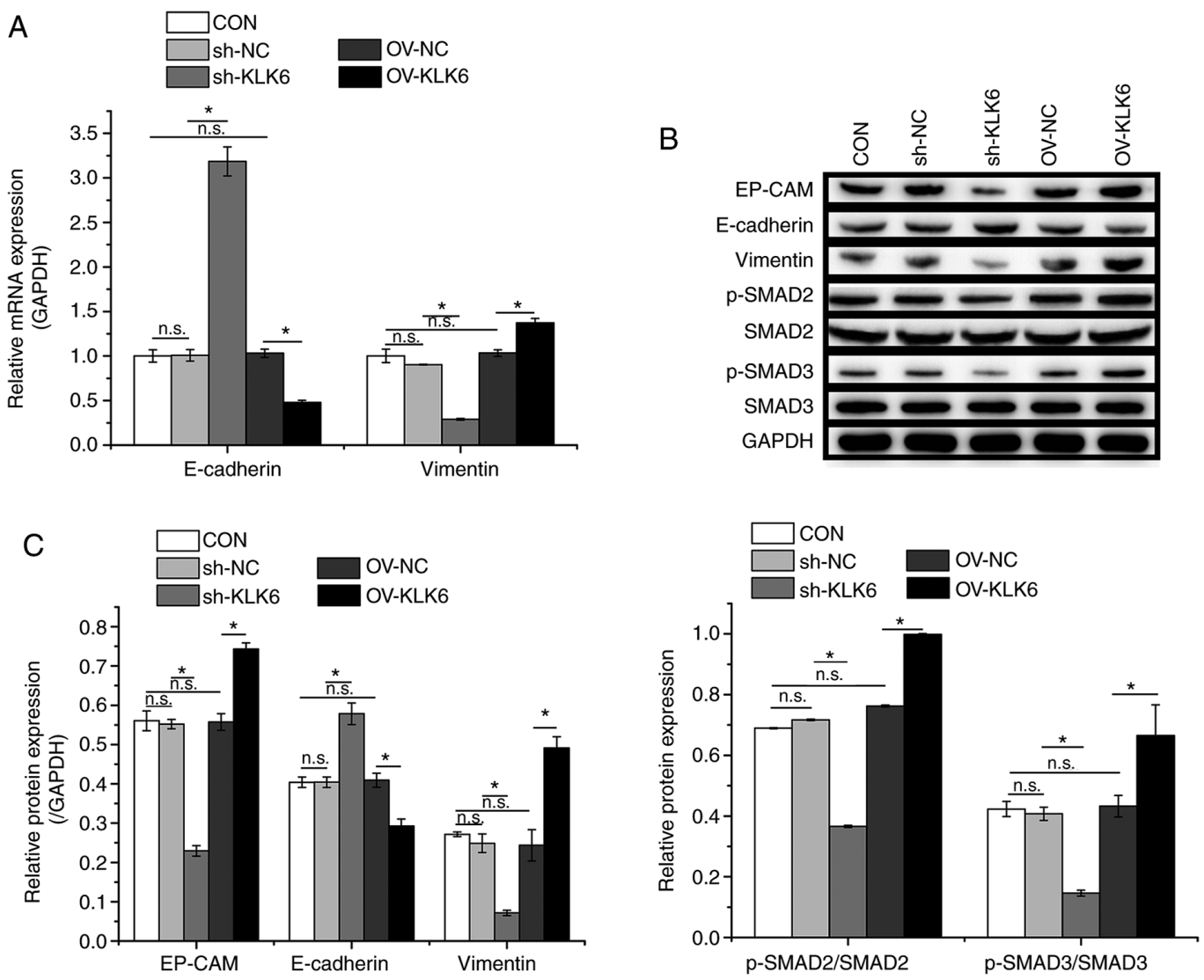

Figure 4. KLK6 inhibition suppresses EMT in HGC-27 cells. (A) Relative mRNA expression levels of E-cadherin and vimentin in HGC-27 cells. (B) Relative protein expression levels of EP-CAM, E-cadherin and vimentin and phosphorylation levels of SMAD2 and SMAD3 in HGC-27 cells. (C) Western blotting densitometry values. Data are presented as the mean \pm SD $(n=3)$. KLK6, kallikrein-related peptidase 6; EMT, epithelial-to-mesenchymal transition; E-cadherin, epithelial cadherin; EP-CAM, epithelial cell adhesion molecule; p-, phosphorylated; Con, untreated control; sh, short hairpin; NC, non-coding control; OV, overexpression plasmid; n.s., not significant. " $\mathrm{P}<0.05$.

A
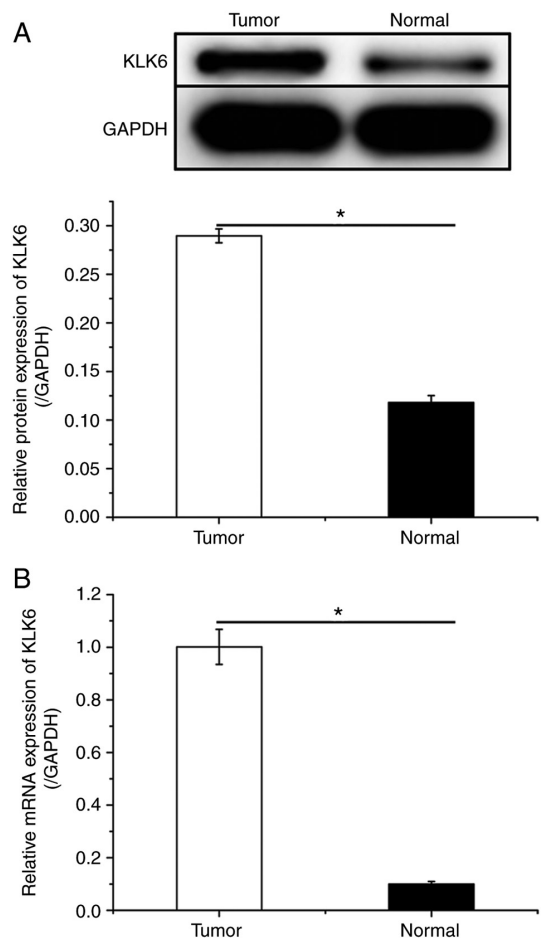

Figure 5. KLK6 is highly expressed in HGC-27-induced xenograft tumor tissue. Relative (A) protein and (B) mRNA expression levels of KLK6 in HGC-27-induced tumor tissue and normal tissue. Data are presented as the mean $\pm \mathrm{SD}(\mathrm{n}=3)$. KLK6, kallikrein-related peptidase 6. ${ }^{*} \mathrm{P}<0.05$. invasion and chemoresistance by regulating EMT (20). KLK6 is also associated with drug resistance in gastric cancer. Kim et al (24) reported that KLK6 expression resulted in chemoresistance by inhibiting auranofin-induced apoptosis via autophagy activation in gastric cancer. In addition, KLK6 has been reported to be highly expressed in gastric cancer (15) and acts as a prognostic indicator for gastric cancer $(25,26)$. Zhu et al (27) found that KLK6 facilitated gastric cancer cell migration, invasion and growth, consistent with the findings of the present study. Additionally, the present study demonstrated that KLK6 enhanced EMT in gastric cancer cells, both in vivo and in vitro, and promoted the progression of gastric cancer cell tumors in vivo.

EMT is a biological process in which epithelial cells transform to adopt a mesenchymal phenotype, resulting in the loss of cell-cell adhesion and cell polarization and in the acquisition of migration and invasion abilities (28). This process occurs during tissue regeneration, organ fibrosis and wound healing (29). EMT has been demonstrated to be a vital mechanism that causes epithelial cancer cells to acquire the migratory and invasive properties associated with metastatic characteristics (30). During EMT, the expression of epithelial markers, including E-cadherin, a type I classical cadherin that plays important roles in intercellular interactions (31), is suppressed. Meanwhile, mesenchymal markers, including vimentin, a cytoskeletal protein involved in regulating cell 

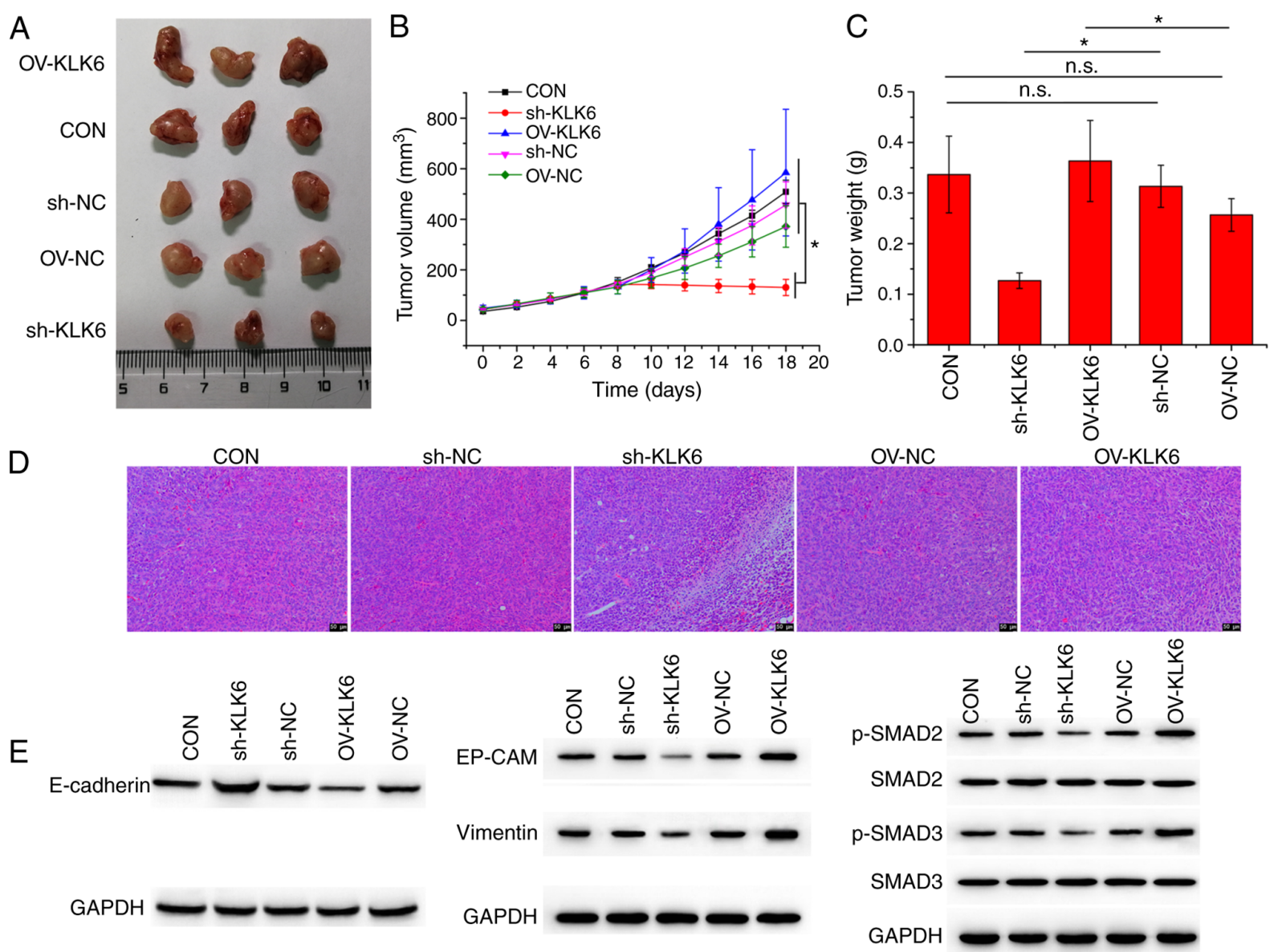

GAPDH

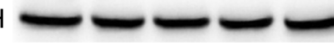

GAPDH

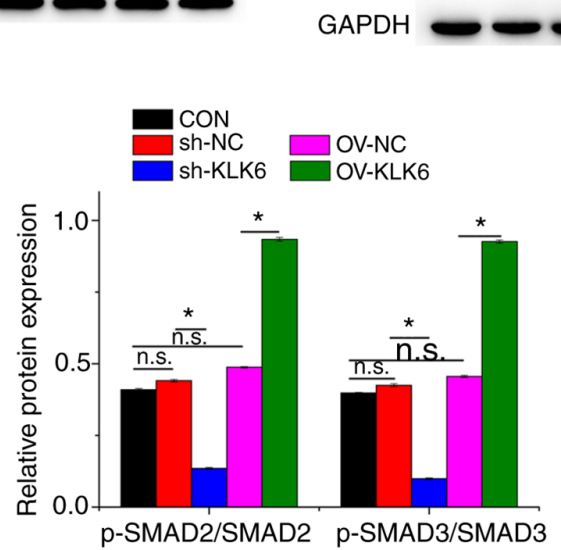

Figure 6. KLK6 inhibition suppresses tumor growth. (A) Representative photographs, (B) volumes and (C) weights of corresponding tumors. (D) Pathological morphology of xenografted tumors. (E) Relative protein expression levels of EP-CAM, E-cadherin and vimentin and levels of phosphorylation of SMAD2 and SMAD3 in xenografted tumors. Data are presented as the mean \pm SD $(n=3)$. KLK6, kallikrein-related peptidase 6; EMT, epithelial-to-mesenchymal transition; E-cadherin, epithelial cadherin; EP-CAM, epithelial cell adhesion molecule; p-, phosphorylated; Con, untreated control; sh, short hairpin; NC, non-coding control; OV, overexpression plasmid; n.s., not significant. "P<0.05.

motility (32), are upregulated (33). The present study revealed that KLK6 interference increased E-cadherin expression and suppressed that of vimentin, suggesting an inhibitory effect of KLK6 on EMT in gastric cancer.

In addition, the present study demonstrated that KLK6 inhibition attenuated the phosphorylation of SMAD2 and SMAD3, both of which are transcription factors that are activated by transforming growth factor- $\beta$ (29). Several studies have reported that SMAD2/3 signaling is associated with EMT. Tang et al (34) revealed that SMAD2/3 activation promoted EMT in lung adenocarcinoma. Kim et al (35) suggested that epidermal growth factor enhanced the phosphorylation of SMAD2/3, thereby inducing EMT in breast cancer cells. Wang et al (36) indicated that microfibril-associated protein 2 promotes EMT in gastric cancer by activating the SMAD2/3 signaling pathway. In addition, it was previously demonstrated that KLK6 activation altered the expression of EMT markers by promoting SMAD2/3 phosphorylation (11). These findings suggest that the effect of KLK6 on EMT in gastric cancer may be associated with the regulation of SMAD2/3 signaling.

In conclusion, the present study demonstrated that KLK6 is involved in the modulation of gastric cancer cell proliferation, migration and invasion via EMT regulation. The effect of KLK6 on EMT in gastric cancer is possibly mediated by 
SMAD2/3 signaling. Whether there are other factors involved in the mechanism of KLK6 in EMT regulation remains to be further elucidated. A limitation of the present study is that only three nude mice were used in each group for the in vivo studies, which may have affected the statistical analysis. However, all animal experiments were performed based on the findings of a preliminary experiment. More animals (at least 6 in each group) will be included in follow-up study designs, which will focus on investigating the effect of KLK6 on EMT-associated metabolic reprogramming in gastric cancer. The lack of co-immunoprecipitation experiments for validating the signaling pathway and the use of only one cell line are other limitations of this study, which will be iresolved in the follow-up study. Overall, the present findings suggest that KLK6 may be a future target for gastric cancer therapy.

\section{Acknowledgements}

Not applicable

\section{Funding}

No funding was received.

\section{Availability of data and materials}

The datasets used and/or analyzed during the present study are available from the corresponding author on reasonable request.

\section{Authors' contributions}

DZ was responsible for the study design, as well as drafting and revision of the manuscript. YH, HL and $\mathrm{WH}$ performed the experiments. YH analyzed the data. DZ and YH were responsible for confirming the authenticity of raw data. All authors read and approved the final manuscript.

\section{Ethics approval and consent to participate}

All animal procedures were approved by the Ethics Committee of Wuhan Myhalic Biotechnology Co., Ltd. (approval no. HLK-20181102-01).

\section{Patient consent for publication}

Not applicable.

\section{Competing interests}

The authors declare that they have no competing interests.

\section{References}

1. Seo GH, Kang HY and Choe EK: Osteoporosis and fracture after gastrectomy for stomach cancer: A nationwide claims study. Medicine (Baltimore) 97: e0532, 2018.

2. Bray F, Ferlay J, Soerjomataram I, Siegel RL, Torre LA and Jemal A: Global cancer statistics 2018: GLOBOCAN estimates of incidence and mortality worldwide for 36 cancers in 185 countries. CA Cancer J Clin 68: 394-424, 2018.

3. Omran AR: The epidemiologic transition: A theory of the epidemiology of population change. 1971. Milbank Q 83: 731-757, 2005.
4. Tian Y, Li X, Li H, Lu Q, Sun G and Chen H: Astragalus mongholicus regulate the Toll-like-receptor 4 meditated signal transduction of dendritic cells to restrain stomach cancer cells. Afr J Tradit Complement Altern Med 11: 92-96, 2014.

5. Feng RM, Zong YN, Cao SM and Xu RH: Current cancer situation in China: Good or bad news from the 2018 Global Cancer Statistics? Cancer Commun (Lond) 39: 22, 2019.

6. Gou WF, Yang XF, Shen DF, Zhao S, Liu YP, Sun HZ, Takano Y, Su RJ, Luo JS and Zheng HC: The roles of BTG3 expression in gastric cancer: A potential marker for carcinogenesis and a target molecule for gene therapy. Oncotarget 6: 19841-19867, 2015.

7. Anisowicz A, Sotiropoulou G, Stenman G, Mok SC and Sager R: A novel protease homolog differentially expressed in breast and ovarian cancer. Mol Med 2: 624-636, 1996.

8. Yang F, Hu ZD, Chen Y and Hu CJ: Diagnostic value of KLK6 as an ovarian cancer biomarker: A meta-analysis. Biomed Rep 4: 681-686, 2016

9. Ghosh MC, Grass L, Soosaipillai A, Sotiropoulou G and Diamandis EP: Human kallikrein 6 degrades extracellular matrix proteins and may enhance the metastatic potential of tumour cells. Tumour Biol 25: 193-199, 2004.

10. Klucky B, Mueller R, Vogt I, Teurich S, Hartenstein B, Breuhahn K, Flechtenmacher C, Angel $P$ and Hess J: Kallikrein 6 induces E-cadherin shedding and promotes cell proliferation, migration, and invasion. Cancer Res 67: 8198-8206, 2007.

11. Chen H, Sells E, Pandey R, Abril ER, Hsu CH, Krouse RS, Nagle RB, Pampalakis G, Sotiropoulou G and Ignatenko NA: Kallikrein 6 protease advances colon tumorigenesis viainduction of the high mobility group A2 protein. Oncotarget 10: 6062-6078, 2019.

12. Pampalakis G, Prosnikli E, Agalioti T, Vlahou A, Zoumpourlis V and Sotiropoulou G: A tumor-protective role for human kallikrein-related peptidase 6 in breast cancer mediated by inhibition of epithelial-to-mesenchymal transition. Cancer Res 69: 3779-3787, 2009.

13. Henkhaus RS, Gerner EW and Ignatenko NA: Kallikrein 6 is a mediator of K-RAS-dependent migration of colon carcinoma cells. Biol Chem 389: 757-764, 2008.

14. Liu X, Xiong H, Li J, He Y and Yuan X: Correlation of hK6 expression with tumor recurrence and prognosis in advanced gastric cancer. Diagn Pathol 8: 62, 2013.

15. Kim JJ, Kim JT, Yoon HR, Kang MA, Kim JH, Lee YH, Kim JW, Lee SJ, Song EY, Myung PK, et al: Upregulation and secretion of kallikrein-related peptidase 6 (KLK6) in gastric cancer. Tumour Biol 33: 731-738, 2012.

16. Livak KJ and Schmittgen TD: Analysis of relative gene expression data using real-time quantitative PCR and the 2(-Delta Delta C(T)) Method. Methods 25: 402-408, 2001.

17. Tang H, Xu L, Cen X, Yang L, Feng J, Li G, Zhu H, Gao S, $\mathrm{Yu} \mathrm{Y}, \mathrm{Zhao} \mathrm{Y}$, et al: $\mathrm{CDK} 5$ inhibition in vitro and in vivo induces cell death in myeloma and overcomes the obstacle of bortezomib resistance. Int J Mol Med 45: 1661-1672, 2020.

18. Ahmed N, Dorn J, Napieralski R, Drecoll E, Kotzsch M, Goettig P, Zein E, Avril S, Kiechle M, Diamandis EP, et al: Clinical relevance of kallikrein-related peptidase 6 (KLK6) and 8 (KLK8) mRNA expression in advanced serous ovarian cancer. Biol Chem 397: 1265-1276, 2016.

19. Talieri M, Zoma M, Devetzi M, Scorilas A and Ardavanis A: Kallikrein-related peptidase 6 (KLK6)gene expression in intracranial tumors. Tumour Biol 33: 1375-1383, 2012.

20. Schrader CH, Kolb M, Zaoui K, Flechtenmacher C, Grabe N, Weber KJ, Hielscher T, Plinkert PK and Hess J: Kallikrein-related peptidase 6 regulates epithelial-to-mesenchymal transition and serves as prognostic biomarker for head and neck squamous cell carcinoma patients. Mol Cancer 14: 107, 2015.

21. Ohlsson L, Lindmark G, Israelsson A, Palmqvist R, Öberg A, Hammarström ML and Hammarström S: Lymph node tissue kallikrein-related peptidase 6 mRNA: A progression marker for colorectal cancer. Br J Cancer 107: 150-157, 2012.

22. Wang P, Magdolen V, Seidl C, Dorn J, Drecoll E, Kotzsch M, Yang F, Schmitt M, Schilling O, Rockstroh A, et al: Kallikrein-related peptidases 4, 5, 6 and 7 regulate tumour-associated factors in serous ovarian cancer. Br J Cancer 119: 1-9, 2018.

23. Krenzer S, Peterziel H, Mauch C, Blaber SI, Blaber M, Angel P and Hess J: Expression and function of the kallikrein-related peptidase 6 in the human melanoma microenvironment. J Invest Dermatol 131: 2281-2288, 2011. 
24. Kim TW, Lee SJ, Kim JT, Kim SJ, Min JK, Bae KH, Jung H, Kim BY, Lim JS, Yang Y, et al: Kallikrein-related peptidase 6 induces chemotherapeutic resistance by attenuating auranofin-induced cell death through activation of autophagy in gastric cancer. Oncotarget 7: 85332-85348, 2016.

25. Nagahara H, Mimori K, Utsunomiya T, Barnard GF, Ohira M, Hirakawa K and Mori M: Clinicopathologic and biological significance of kallikrein 6 overexpression in human gastric cancer. Clin Cancer Res 11: 6800-6806, 2005.

26. Kolin DL, Sy K, Rotondo F, Bassily MN, Kovacs K, Brezden-Masley C, Streutker CJ and Yousef GM: Prognostic significance of human tissue kallikrein-related peptidases 6 and 10 in gastric cancer. Biol Chem 395: 1087-1093, 2014.

27. Zhu S, Shi J, Zhang S and Li Z: KLK6 Promotes Growth, Migration, and Invasion of Gastric Cancer Cells. J Gastric Cancer 18: 356-367, 2018

28. Gloushankova NA, Zhitnyak IY and Rubtsova SN: Role of Epithelial-Mesenchymal Transition in Tumor Progression. Biochemistry (Mosc) 83: 1469-1476, 2018.

29. Liang X, He X, Li Y, Wang J, Wu D, Yuan X, Wang X and Li G: Lyn regulates epithelial-mesenchymal transition in CS-exposed model through Smad2/3 signaling. Respir Res 20: 201, 2019.

30. Sun L and Fang J: Epigenetic regulation of epithelial-mesenchymal transition. Cell Mol Life Sci 73: 4493-4515, 2016.
31. Bure IV, Nemtsova MV and Zaletaev DV: Roles of E-cadherin and Noncoding RNAs in the Epithelial-mesenchymal Transition and Progression in Gastric Cancer. Int J Mol Sci 20: 20, 2019.

32. Wang N, Liu D, Guo J, Sun Y, Guo T and Zhu X: Molecular mechanism of Poria cocos combined with oxaliplatin on the inhibition of epithelial-mesenchymal transition in gastric cancer cells. Biomed Pharmacother 102: 865-873, 2018.

33. Cevenini A, Orrù $S$, Mancini A, Alfieri $A$, Buono $P$ and Imperlini E: Molecular Signatures of the Insulin-like Growth Factor 1-mediated Epithelial-Mesenchymal Transition in Breast, Lung and Gastric Cancers. Int J Mol Sci 19: 19, 2018.

34. Tang Y, Xuan Y, Qiao G, Ou Z, He Z, Zhu Q, Liao M and Yin G: MDM2 promotes epithelial-mesenchymal transition through activation of Smad2/3 signaling pathway in lung adenocarcinoma. OncoTargets Ther 12: 2247-2258, 2019.

35. Kim J, Kong J, Chang H, Kim H and Kim A: EGF induces epithelial-mesenchymal transition through phospho-Smad2/3-Snail signaling pathway in breast cancer cells. Oncotarget 7: 85021-85032, 2016.

36. Wang JK, Wang WJ, Cai HY, Du BB, Mai P, Zhang LJ, Ma W, Hu YG, Feng SF and Miao GY: MFAP2 promotes epithelial-mesenchymal transition in gastric cancer cells by activating TGF- $\beta /$ SMAD2/3 signaling pathway. OncoTargets Ther 11: 4001-4017, 2018 